\title{
Sentido de coherencia y salud en personas adultas mayores autopercibidas como sanas
}

\section{Sense of coherence and health in self-perceived healthy aged people}

\author{
Zoila Edith Hernández Zamora ${ }^{a}$, Yamilet Ehrenzweig Sánchez ${ }^{\mathrm{b}}$ y Liliana Yépez Olvera ${ }^{*} *$ \\ Universidad Veracruzana, México
}

(recibido 15 de diciembre de 2010; aceptado 27 de Mayo de 2011)

\begin{abstract}
Resumen
Este trabajo tiene como objetivo principal identificar la relación entre el nivel de sentido de coherencia de una muestra de adultos mayores de una estancia diurna de la ciudad de Xalapa, Veracruz, México, con la autopercepción que tienen de su salud, así como el estado de salud real con que cuentan en el momento actual. Para lo cual se aplicó un cuestionario de datos generales, el Cuestionario de Orientación hacia la Vida de Antonovsky y el Cuestionario General de Salud de Goldberg. Los resultados muestran un alto sentido de coherencia así como un buen nivel de salud mental en la mayoría de estas personas.
\end{abstract}

Palabras clave: adultos mayores, salud, sentido de coherencia, salud mental, estilos de vida

\begin{abstract}
This paper aims to identify the relationship between the level of sense of coherence in a sample of elderly people at a daycare facility in the Mexican city of Xalapa, in the state of Veracruz, and the self-perception that they manifest about their own health status, as well as their real state of health. To this end, two surveys were conducted: Antonovsky's Questionnaire of Orientation towards Life, which includes general data items, and Goldberg's General Health Questionnaire. The results show a high sense of coherence, as well as a good level of mental health, in most of the aged people who participated in the sample.
\end{abstract}

Keywords: elderly people, aged people, health, sense of coherence, mental health, lifestyles

* Instituto de Investigaciones Psicológicas de la Universidad Veracruzana, Dr. Luis Castelazo Ayala s/n, Col. Industrial Ánimas, 91190, Xalapa, Ver., México. Correspondencia. Correos electrónicos:

a_zhernandez@uv.mx;

byehrenzweig@uv.mx;

c lili_yepez_olvera@yahoo.com.mx

ISSN 0257-1439

(C) Colegio Profesional de Psicólogos de Costa Rica

http://www.revistacostarricensedepsicologia.com/ 
Según Ashton y Seymour (1990, citados en Gallard, 2002) existen factores determinantes en la salud de las personas, mismos que son definidos como los elementos que delimitan las condiciones de salud de un individuo o comunidad. La promoción de la salud pretende capacitar a los individuos de una comunidad para que ellos controlen sus determinantes de salud y, puedan así mejorarla. Estos autores consideran que los principales determinantes de salud son: dotación genética, factores ambientales, factores nutricionales, salud laboral y estilos de vida.

Además de estos factores, se hace necesario mencionar el aspecto social, donde el entorno familiar y de las demás personas que rodean al individuo juegan un papel fundamental en su bienestar emocional o psicológico, ambos, tanto apoyo social como bienestar psicológico son factores también determinantes de la salud.

Según Giraldo, Toro, Macías, Valencia y Palacios (2010), en el mantenimiento de la salud intervienen cuatro factores fundamentales en diversas proporciones: factores biológicos individuales determinados por la carga genética (25\%), medio ambiente $(15 \%)$, atención médica (10\%) y estilos de vida, actitudes y comportamiento (50\%).

De los factores anteriormente mencionados, como puede observarse, los que tienen un mayor impacto en la salud de las personas son los estilos de vida, mismos que según Last (1998, citado en Aburto, 2001, p. 54) son:

El conjunto de hábitos y costumbres que es influido, modificado, estimulado o restringido por el proceso de socialización de toda la vida. Estos hábitos y costumbres incluyen el uso de sustancias como el alcohol, tabaco, té, café, hábitos dietéticos, ejercicio, conductas sexuales, entre otros, que tienen importantes implicaciones para la salud y son frecuentemente el tópico de investigaciones epidemiológicas.

En complemento, los estilos de vida saludables son definidos como los procesos sociales, las tradiciones, los hábitos, conductas y comportamientos de los individuos y grupos de población que conllevan a la satisfacción de las necesidades humanas para alcanzar el bienestar y la vida (Duchan \& Black, 2001).

Los estilos de vida son determinados por la presencia de factores de riesgo o de factores protectores para el bienestar, por lo cual deben ser vistos como un proceso dinámico que no sólo se compone de acciones o comportamientos individuales, sino también de acciones de naturaleza social (Meda, Torres, Cano, \& Vargas, 2004).

En esta forma se puede elaborar una serie de estilos de vida saludables o comportamientos saludables o factores protectores de la calidad de vida, que al asumirlos responsablemente ayudan a prevenir desajustes biopsicosociales y mantener el bienestar para generar calidad de vida, satisfacción de necesidades y desarrollo humano.

La estrategia para desarrollar estilos de vida saludables es el compromiso individual y social, ya que éste es básico para satisfacer las necesidades fundamentales, se mejora la calidad de vida y se alcanza el desarrollo humano en términos de la dignidad de la persona (Meda et al., 2004).

Los estilos de vida saludables están íntimamente relacionados con las conductas de salud, que son aquellas que las personas adoptan en ausencia de cualquier indicio o síntoma, y que están destinadas a mantenerse bien o a incrementar el estado de bienestar. Ejemplos de tales conductas serían vacunarse, hacer ejercicios, realizar exámenes médicos preventivos, usar cinturón de seguridad, tomar vitaminas y seguir una dieta equilibrada, entre otras. Es decir, se refieren a 
acciones que realizamos mientras estamos saludables y que pueden mantener o mejorar nuestro estado de salud (Barra, 2002).

En cuanto a la calidad de vida, el nivel en que ésta se encuentre en una persona estará fuertemente influida por sus estilos de vida. En 1998, Sen presenta su enfoque de calidad de vida centrado en las capacidades que permiten funcionar en la vida: poder hacer y ser, argumentando la diferencia entre el capital humano y la capacidad humana (Sen, 2004) y hace referencia al funcionamiento que significan las partes del estado de una persona, en particular las cosas que logra hacer o ser en su vida.

Para el estudio de la calidad de vida es importante también considerar el plano subjetivo de las capacidades, explicadas como capacidades intelectuales y emotivas que la educación y la socialización proporcionan (Suárez, Del Toro, Moncada, Vinent, \& Peña, 2001) y que son referidas como habilidades mentales y sociales de adaptación a las circunstancias de la vida que el individuo posee o desarrolla (Gil-Monte \& Peiró, 1997; Lazarus \& Folkman, 1991) y que al ser puestas en acción resultan en la salud física, mental y social o en el bienestar personal.Lo anterior está en relación al "querer hacer" limitado por el "poder hacer", en que las actividades, que crean y elevan las determinantes perdurables de la acción (las capacidades) y que impactan las experiencias presentes y futuras, fortalecen el valor de la inversión, capitalizan al individuo, lo fortalecen en lo que puede hacer y, más importante aún, en lo que cree poder hacer y ser.

Este razonamiento pone el énfasis en la subjetividad sin descuidar lo objetivo, y puede referirse a los aspectos particulares de las capacidades con que los individuos creen contar, poniendo en juego las habilidades en el quehacer cotidiano al enfrentar los retos de la vida (Arita \& Herrán de la, 2007; Herrán de la \& Atrita, 2003). Como resultado de lo anterior, la evaluación de este proceso de vida se refleja en el bienestar subjetivo mediante la satisfacción y felicidad sentidas (Arita, 2006; Diener \& Suh, 2001).

Frente a situaciones de adversidad tales como catástrofes naturales o sociales, así como eventos vitales altamente perturbadores, es habitual en Psicología cuestionar acerca del impacto negativo que estas situaciones pueden tener sobre la salud psíquica de un individuo que ha estado expuesto a tales escenarios. Sin embargo, parece igual de importante e interesante poder situar, qué sucede con aquellas personas que sobrellevan estas situaciones sin evidenciar necesariamente consecuencias por la vía sintomática o psicopatológica. Estas personas parecieran disponer de recursos subjetivos tales que permitirían amortiguar el impacto de esos eventos, así como dar curso más elaborado al dolor psíquico que situaciones semejantes pudieran conllevar (Brizzio \& Carreras, 2007).

Por otro lado, también es posible afirmar que la cotidianidad misma demanda demasiados esfuerzos para sobrellevar distintas situaciones perturbadoras. Eventos que pueden ser considerados mínimos e insignificantes por ciertos observadores, pueden desencadenar situaciones de suma angustia por otros menos resguardados. Es decir, que tanto escenas masivas como íntimas, esporádicas como cotidianas, pueden convertirse en situaciones de adversidad, merecedoras de esfuerzos subjetivos de superación. 
Frente a este escenario, Antonovsky $(1979,1987)$, sociólogo, investigador de la Universidad de Ben Gurion, Israel, se preguntó:

¿Qué es aquello que nos mantiene sanos, a pesar de que estemos sometidos a la continua influencia de condiciones ambientales adversas? ¿Por qué algunas personas muestran dificultades para superar situaciones conflictivas y otras, que atravesaron las mismas circunstancias vitales, resultan indemnes a tal situación?

Este autor dirigió sus investigaciones hacia el estudio de la relación entre situaciones, fenómenos o eventos vitales estresantes y la salud psíquica. Es decir, qué es aquello que hace que la exposición frente distintos tipos de sucesos de vida perturbadores pueda ser tramitada o elaborada, de modo tal, de no presentar consecuencias sintomáticas.

Antonovsky (1979) propone, entonces, un marco para el estudio de la temática denominada orientación salugénica, de ahí el nombre que se emplea para caracterizar a las variables como salugénicas, utilizando el término salud (del lat. salus) y origen (del lat. génesis).

Este modelo de abordaje propone orientar las investigaciones en el campo de la ciencia de la salud, centrándose en los recursos, así como en las disposiciones personales, que permiten amortiguar el impacto de los estímulos externos e internos para buscar salidas alternativas frente al dolor psíquico, que no sean patológicas. Estos estímulos estresantes pueden conformar un evento, suceso o circunstancia de la vida de una persona, capaz de alterar o modificar sus condiciones de salud psicofísica (Casullo, 2008).

Antonovsky (1987) plantea este tipo de estudio, en cierta medida oponiéndose a una visión patogénica, descrita como aquello que está directa y específicamente orientada a descubrir las causas de la enfermedad de las personas y el desarrollo de trastornos y estructuras patológicas particulares. Este conocimiento se utiliza para luego poder encontrar formas de prevenir y combatir estas enfermedades. En el modelo patogénico se asume que las causas de la enfermedad son agentes físicos, bioquímicos, microbiológicos y psicosociales, o más precisamente, en la determinación multifactorial de las enfermedades, usualmente, en términos de factores de riesgo. Desde ese paradigma puede pensarse que la exposición a situaciones o eventos estresantes pueden conformar un factor de riesgo para contraer enfermedad o padecimiento.

Sin embargo, para Antonovsky (1987) eso no es así, por el contrario, suponer que la exposición a situaciones de estrés conlleva inevitablemente la presencia de sintomatología psicopatológica, es errónea. El autor hace hincapié en la elaboración de esas situaciones y las distintas salidas posibles frente a un hecho o estímulo determinado, es decir, que no se trata de evitar el impacto subjetivo percibido a partir de determinada escena, aunque sí, tal vez, una mayor elaboración de lo acontecido, producto de una disposición personal particular, permita disminuir ese impacto, de modo tal, que redunde en salidas más saludables.

A lo largo de la conceptualización de esta orientación, se vislumbra la intención del autor de especificar alguna respuesta frente a la pregunta salugénica: ¿qué es lo que nos mantiene orientados hacia el polo saludable del continuum salud-enfermedad? Esta respuesta toma forma en el desarrollo que el autor construye del concepto que denomina sentido de coherencia (SOC), que conforma la disposición personal que permite a las personas lograr esta inclinación saludable.

Antonovsky (1987) define el SOC como la orientación global de un individuo que expresa el grado en que posee sentimientos de confianza penetrantes, estables y dinámicos, acerca de que: 
(a) los estímulos que derivan del medio interno o externo en el curso de la vida, son estructurables, predecibles y explicables, (b) los recursos para responder a las demandas estimulares están disponibles; (c) estas demandas son desafíos en los que resulta importante invertir y comprometerse (p. 19).

El SOC es una disposición u orientación personal, no un estado o un rasgo; tampoco es una estrategia de afrontamiento en sí misma. Sino que aquellas personas que lo poseen de manera notable, sabrán llevar a cabo estrategias más apropiadas para afrontar las demandas estimulares (Antonovsky, 1992).

Para Antonovsky (1987), una persona con alto sentido de coherencia experimenta menos emociones negativas, tales como depresión y ansiedad, pudiendo hacer frente a los estímulos estresantes de un modo más elaborado, redundando en una mayor percepción subjetiva de bienestar psicológico. El sentido de coherencia y el bienestar psicológico son dos variables salugénicas.

En cuanto a la salud de las personas mayores, grupo etario que nos ocupa en este trabajo, existen enormes diferencias en la manera en que cada persona envejece, que van desde las habilidades físicas hasta la energía y el compromiso con la vida.

Sin embargo, en el modelo de la curva normal de maduración aparecen modificaciones predecibles durante el desarrollo de una persona. Asociados a la edad, estos cambios suelen formularse como un movimiento ascendente que implica el aumento de capacidades y habilidades hasta un periodo más o menos predeterminado, que se define a través de algunos tests, pruebas y mediante observaciones del desenvolvimiento de los sujetos en la vida cotidiana (Muchinik, 2006)

Para Birren (1996) la edad cronológica es como ver la vida en blanco y negro, solamente un criterio clasificatorio, según el cual sería posible definir el curso paralelo de los procesos de maduración y deterioro y las pautas de conducta que de ellos se desprenden. La asignación de rasgos atribuibles a los senectos surge de utilizar estos criterios para caracterizar a los mismos.

Aunque no está a nuestro alcance el modificar el proceso de envejecimiento, hoy sabemos de él mucho más que hace apenas unas décadas. ¿Qué es el envejecimiento físico? Un estudio longitudinal de los que marcan época está revelando el misterio:

El estudio longitudinal de Baltimore (Belsky, 2001) sobre el envejecimiento, que se empezó en 1959 constituye el mejor esfuerzo realizado para investigar el envejecimiento físico en carne y hueso. En este ambicioso estudio, varias personas voluntarias pasan varios días en el Centro de Investigaciones Gerontológicas de Baltimore una vez al año, o quizá cada dos años, para que se les pueda comprobar y examinar cualquier posible función física.

El estudio de Baltimore demuestra que no podemos pensar en el envejecimiento físico únicamente desde el punto de vista de la pérdida. En esta importante fase de la vida existe la estabilidad. Incluso cuando los años se cobran un peaje físico, nuestro cuerpo sabe recuperarse. Tenemos la capacidad de crecer y adaptarnos hasta en áreas como el cerebro, donde los científicos no habían podido predecir un crecimiento después de la madurez.

La expectativa de vida aumenta globalmente en el mundo debido a varios factores como los nutricionales, los socioculturales y los económicos. Alrededor del $42 \%$ de los hogares tienen al menos un adulto mayor (Cornachione, 2006), por lo tanto, se hace necesario que esta población, que cada día ocupa un mayor número en nuestra sociedad, no sólo viva más años, sino que lo haga de manera saludable. 
La finalidad de esta investigación es pues, estudiar personas sanas, desde el modelo de la salud, a través de su sentido de coherencia, y no desde el de la enfermedad, ya que la mayoría de las investigaciones sobre este grupo etario presentan individuos atípicos, enfermos, recluidos en instituciones, ya sea en asilos, hospitales o estancias geriátricas. El estudio se llevará a cabo con personas sanas, o bien, que se perciban como sanas, que llevan una vida dentro de sus hogares, generalmente con su familia, que hacen al menos una actividad que les gusta y que no padecen enfermedades que los incapaciten para disfrutar de una buena calidad de vida. Se trata de investigar lo que ellos han hecho para tener un envejecimiento saludable.

\section{Método}

\section{Muestra}

Se trabajó con una muestra intencional voluntaria de 67 adultos mayores, o sea, personas de más de 60 años, 19 hombres y 48 mujeres, con una edad promedio de 69.3 años, $46.3 \%$ con estudios profesionales y $23.9 \%$ con bachillerato, $44.8 \%$ amas de casa y $22.4 \%$ jubilados o pensionados, aunque hay que aclarar que nueve de estas amas de casa eran jubiladas o pensionadas; $55.2 \%$ con pareja. El criterio de inclusión es que tuvieran 60 o más años de edad y desearan participar en el estudio, además de que percibieran su salud como buena o muy buena. Los cuestionarios fueron aplicados de manera individual en la modalidad de entrevista.

\section{Muestreo}

La selección de los participantes se hizo mediante un muestreo por oportunidad, el cual consiste en seleccionar a aquellos sujetos voluntarios y que presentan mayor oportunidad de ser seleccionados (Coolican, 1994). La investigación se llevó a cabo a través de un diseño descriptivo de tipo transversal.

\section{Situación o ámbito}

El estudio se llevó a cabo en una estancia diurna que depende del DIF (Desarrollo Integral de la Familia) estatal y que es exclusiva para el uso de personas mayores de 60 años. Está compuesta por clubes que se agrupan dependiendo de las intereses de las personas, y se reúnen de una a tres veces la semana.

\section{Instrumentos}

Cuestionario de datos generales: Se aplicó un cuestionario de datos generales donde se recabó información sobre edad, sexo, escolaridad, enfermedades, estado de salud percibido, ocupación, estado civil, satisfacción con el apoyo social recibido, estado de ánimo, entre otras. Además de identificar los principales comportamientos que la población de estudio considera han contribuido para mantener una buena salud.

Cuestionario de Orientación hacia la Vida (Orientation to Life Questionnaire, OLQ, Sense of Coherence, SOC, Antonovsky, 1987). Sentido de coherencia (SOC) fue evaluado usando el OLQ, el cual consta de tres subescalas: comprensibilidad, manejabilidad y significado.

En este estudio se ha tomado la versión original que consta de 29 ítems. El rango de respuesta de los ítems es de 1 a 5 en escala tipo Likert. Estudios previos demuestran que el cuestionario cumple los criterios de fiabilidad test-retest (Coe, Romers, Tang, \& Wolinsky, 1990; Radmacher \& Sheridan, 1989) y validez (Carmel, Anson, Levenson, Bonneth, \& Maoz, 1991; Margalit, Leyser, \& Avraham, 1988). Antonovsky (1987) aporta índices de consistencia interna entre .84 y .93. 
El sentido de coherencia es un patrón cognitivo-motivacional o una orientación global en la forma de ver las situaciones de la vida. Antonovsky refiere que puede guardar relación con la salud influyendo en el proceso de evaluación que una persona hace de las situaciones como estresantes o no, moderando el grado de tensión que un estresor produce y moderando también las consecuencias adversas para la salud.

Para evaluar el nivel de sentido de coherencia de cada una de las personas entrevistadas, se obtuvo el puntaje máximo que pudieran obtener las personas así como el mínimo, dividiéndose de manera proporcional para obtener así tres niveles en los cuales ubicarlos: alto, medio y bajo sentido de coherencia. Aunque, como puede observarse más adelante, en la tabla correspondiente, no hubo personas con puntajes bajos, lo cual provocó que sólo hubiera niveles medio y alto de sentido de coherencia en las personas de la muestra.

Cuestionario de Salud General (General Health Questionnaiure, GHQ, Goldberg, 1996). En su versión abreviada (12 preguntas) es un instrumento estandarizado para México y se ha utilizado dentro de los programas de atención primaria a la salud o para evaluar a pacientes de consulta externa en el servicio de medicina general, situaciones donde ha sido ampliamente validado para su uso en la población mexicana (Caraveo, González, \& Ramos, 1985; Ezban, Padilla, Medina, \& Gutiérrez, 1985; Medina, Padilla, \& Ezban, 1983).

Se trata de un cuestionario que, además de servir para la detección de casos probables de trastornos psiquiátricos, permite conocer las actitudes del sujeto en cuanto a su estado de salud y para identificar la aparición de fenómenos asociados con situaciones de distrés natural como serían depresión, ansiedad, deterioro social y trastornos somáticos (Ezban et al., 1985; Mc Dowell \& Newell, 1987; Medina et al., 1983; Romero \& Medina, 1987).

El GHQ consta de 12 reactivos en su versión breve, que la persona debe contestar a partir de identificar las sensaciones que sobre su estado de salud ha percibido en el último mes. Los reactivos deben ser contestados en cada una de las cuatro opciones que se les presentan, que van de la letra A a la D. En la mayoría de las ocasiones, las preguntas van de más a menos en la intensidad de los síntomas. El contenido de un reactivo se considera problema cuando el sujeto contesta a las opciones C o D. Para calificarlo, se utiliza una escala de tipo Likert donde a las opciones se les da un puntaje de 0-0-1-1, correspondientes a A, B, C y D.

Este test, además de servir de tamizaje a problemas psiquiátricos, permite conocer las percepciones del sujeto en cuanto a su estado de salud en general. Ha sido considerado por algunos autores como instrumento para evaluar bienestar psicológico, pero se prefiere retomar la intención original del autor, al llamarlo Cuestionario de Salud General o de Salud Mental, como ha sido universalmente aceptado. En realidad, el bienestar psicológico es un determinante de la salud y, a la vez, un componente de ella (por eso se acepta su inclusión en un cuestionario de salud general), pero no son categorías sinónimas. En la literatura revisada se han encontrado autores que emplean el GHQ como indicador de otras categorías tales como "dimensión psicológica de la calidad de vida en el ámbito de salud" (Naughton \& Wiklund, 1993). Pudiera decirse que coinciden con Goldberg al considerarlo como la expresión mental de la salud, o cuestionario de salud mental.

Este cuestionario fue aplicado para detectar personas con posible patología mental y que, en un determinado momento se reportaron como sanas refiriéndose sólo a su estado de salud física. 


\section{Resultados}

Como consecuencia de que la población de estudio se limitó a una muestra en una sola comunidad de adultos mayores, se sugiere no generalizar los resultados a otras comunidades o grupos de adultos mayores. No obstante, los datos recabados ofrecen información de sumo interés y utilidad en la prevención, mantenimiento y mejora de la salud tanto de este grupo etario como de otros grupos de edad.

Como ya se mencionó, debido a que las personas de la muestra obtuvieron altos puntajes en sentido de coherencia, fueron ubicadas, de acuerdo a su puntuación obtenida, en nivel medio y alto, de lo cual se obtiene que el 58 (86.6\%) posee alto nivel de SOC y 9 (13.4\%) un nivel medio. En cuanto a los probables casos psiquiátricos encontrados, sólo se detectaron 6 personas $(9 \%)$, que es un porcentaje relativamente bajo.

En cuanto a los análisis de asociación, se procedió a calcular tablas de contingencia y correlaciones producto-momento (Pearson), definiendo como nivel de significancia aceptable $p<$ .05 (dos colas).

En la tabla 1 se observa que existe una correlación positiva y altamente significativa ( $p<$ .001) entre ser o no caso probable y el nivel de sentido de coherencia (SOC). Asimismo, en la tabla de contingencias se puede observar que, entre las personas con niveles altos de SOC, hubo menos casos probables psiquiátricos, en comparación con aquellas que poseen niveles medios.

\section{Tabla 1}

Tablas de contingencia y correlación Pearson entre nivel de SOC y caso/no caso probable psiquiátrico

\begin{tabular}{cccc|cc|cc}
\hline \multicolumn{9}{c}{ OLQ (SOC) } \\
\hline & \multicolumn{2}{c|}{ Alto 108-145 } & \multicolumn{2}{c}{ Medio 69-107 } & \multicolumn{2}{c}{ Total } \\
\hline \multirow{2}{*}{ CGS } & $n$ & $\%$ & $n$ & $\%$ & $n$ & $\%$ \\
& No caso & 56 & 91.80 & 5 & 8.20 & 61 & 100 \\
& Caso & 2 & 33.30 & 4 & 66.70 & 6 & 100 \\
\hline & Total & 58 & 86.60 & 9 & 13.40 & 67 & 100
\end{tabular}

Nota $:$ CGS = Cuestionario de Salud General; OLQ = Cuestionario de Orientación a la Vida

\begin{tabular}{clcc}
\multicolumn{2}{c}{ Correlación Bivariada } & CGS & OLQ \\
\hline Edad & Pearson Correlation & 1 & $.490 * * *$ \\
& $\mathrm{~N}$ & 67 & 67 \\
\multirow{2}{*}{ OLQ } & Pearson Correlation & $.490^{* * *}$ & 1 \\
& $\mathrm{~N}$ & 67 & 67 \\
\hline Nota: $* * * \mathrm{p}<.001$ (dos colas) &
\end{tabular}


Tabla 2

Tablas de contingencia y correlación Pearson entre nivel de SOC y edad

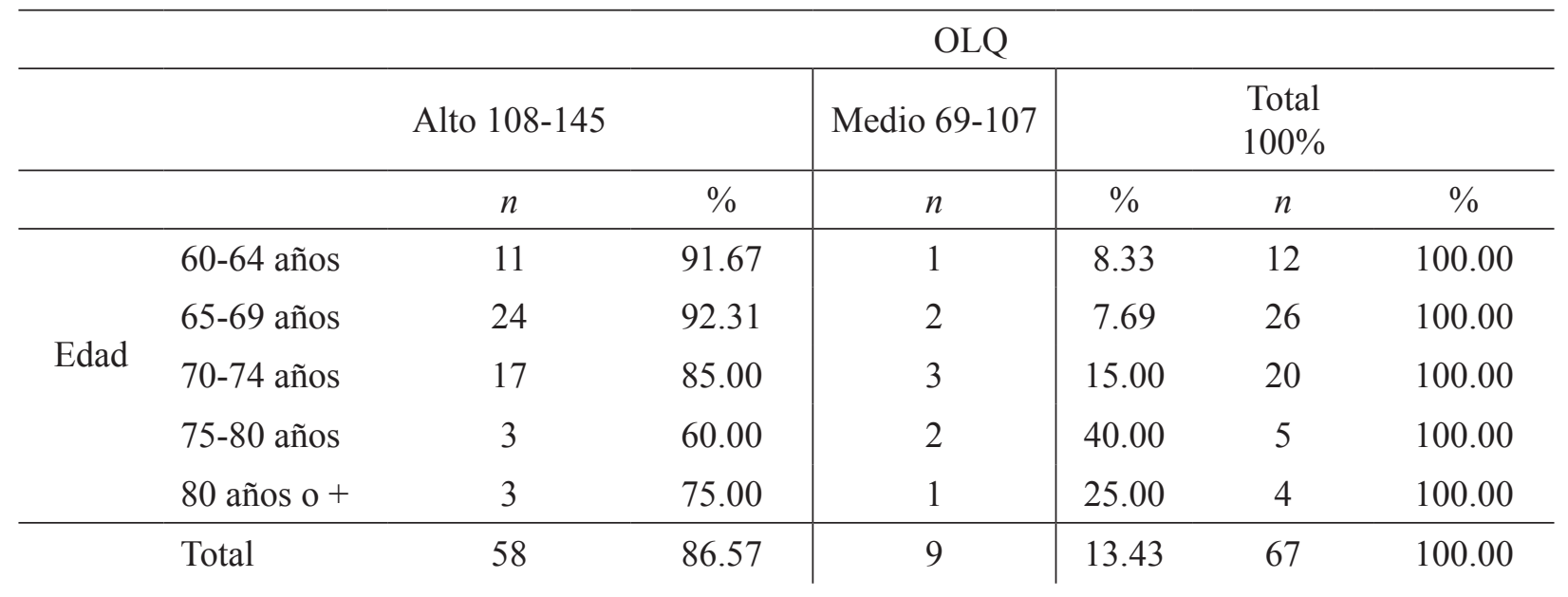

Nota: OLQ $=$ Cuestionario de Orientación a la Vida

\begin{tabular}{clcc} 
& Correlación Bivariada & Edad & OLQ \\
\hline \multirow{3}{*}{ Edad } & Pearson Correlation & 1 & .206 \\
& $\mathrm{~N}$ & 67 & 67 \\
\multirow{3}{*}{ OLQ } & Pearson Correlation & .206 & 1 \\
& $\mathrm{~N}$ & 67 & 67 \\
\hline
\end{tabular}

Al correlacionar edad con SOC (OLQ) se detectó, de acuerdo con la prueba estadística, que no existe relación significativa entre estas variables.

Tabla 3

Tablas de contingencia y correlación Pearson entre nivel de SOC y sexo

\begin{tabular}{cccc|cc|cc}
\hline & \multicolumn{7}{c}{ OLQ } \\
\cline { 3 - 8 } & & Alto & $108-145$ & Medio & $69-107$ & \multicolumn{2}{c}{ Total 100\% } \\
\hline \multirow{2}{*}{ Sexo } & $n$ & $\%$ & $n$ & $\%$ & $n$ & $\%$ \\
\cline { 3 - 8 } & Masculino & 16 & 84.2 & 3 & 15.8 & 19 & 100.00 \\
& Femenino & 42 & 87.5 & 6 & 12.5 & 48 & 100.00 \\
& Total & 58 & 86.6 & 9 & 13.4 & 67 & 100.00 \\
\hline
\end{tabular}

Nota: OLQ $=$ Cuestionario de Orientación a la Vida

(continúa) 
Tabla 3 (continuación)

\begin{tabular}{llcc} 
& Correlación Bivariada & Sexo & OLQ \\
\hline Sexo & Pearson Correlation & 1 & -.043 \\
& $\mathrm{~N}$ & 67 & 67 \\
OLQ & Pearson Correlation & -.043 & 1 \\
& $\mathrm{~N}$ & 67 & 67 \\
\hline
\end{tabular}
SOC.

Según se observa en la tabla 3 , no existe asociación significativa entre sexo y el nivel de Tabla 4

Tabla de contingencia y correlación Pearson entre SOC y número de enfermedades

\begin{tabular}{clcc|cc|cc}
\hline \multicolumn{7}{c}{ OLQ (SOC) } \\
\hline & \multicolumn{2}{c}{ Alto 108-145 } & \multicolumn{2}{c}{ Medio 69-107 } & \multicolumn{2}{c}{ Total } \\
\cline { 2 - 8 } & & $n$ & $\%$ & $n$ & $\%$ & $n$ & $\%$ \\
\hline \multirow{3}{*}{ Número de } & Ninguna & 39 & 88.60 & 5 & 11.40 & 44 & 100.00 \\
enfermedades & Una & 16 & 88.90 & 2 & 11.10 & 18 & 100.00 \\
& Dos & 2 & 66.70 & 1 & 33.30 & 3 & 100.00 \\
& Tres & 1 & 100.00 & 0 & 0.00 & 1 & 100.00 \\
& Cuatro & 0 & 0.00 & 1 & 100.00 & 1 & 100.00 \\
& Total & 58 & 86.60 & 9 & 13.40 & 67 & 100.00 \\
\hline
\end{tabular}

Nota: OLQ $=$ Cuestionario de Orientación a la Vida

\begin{tabular}{llcc}
\multicolumn{1}{c}{$\begin{array}{c}\text { Correlación } \\
\text { Bivariada }\end{array}$} & & Número de enfermedades & OLQ \\
\hline Número de & Pearson Correlation & 1 & .216 \\
enfermedades & $\mathrm{N}$ & 67 & 67 \\
\multirow{2}{*}{ OLQ } & Pearson Correlation & .216 & 1 \\
& $\mathrm{~N}$ & 67 & 67 \\
\hline
\end{tabular}

Si bien todas las personas entrevistadas se percibían como sanas, no por ello estaban, algunas exentas de tener una o más enfermedades. Aunque lo importante de este estudio era su autopercepción, percepción que en la gran mayoría fue de poseer buena salud. Muy probablemente por esto no hubo correlación significativa entre el nivel de sentido de coherencia y número de enfermedades. 
Tabla 5

Tablas de contingencia y correlación Pearson entre el nivel de SOC y qué es lo que ha sido más importante en la vida de los entrevistados

\begin{tabular}{|c|c|c|c|c|c|c|c|}
\hline \multicolumn{8}{|c|}{ OLQ (SOC) } \\
\hline & & \multicolumn{2}{|c|}{ Alto $108-145$} & \multicolumn{2}{|c|}{ Medio 69-107 } & \multicolumn{2}{|r|}{ Total } \\
\hline & & $n$ & $\%$ & $n$ & $\%$ & $n$ & $\%$ \\
\hline \multirow{4}{*}{$\begin{array}{l}\text { ¿Qué ha sido lo } \\
\text { más importante } \\
\text { para usted? }\end{array}$} & Situación afectiva & 33 & $97.10 \%$ & 1 & $2.90 \%$ & 34 & $100.00 \%$ \\
\hline & Situación económica & 2 & $66.70 \%$ & 1 & $33.30 \%$ & 3 & $100.00 \%$ \\
\hline & Su salud & 22 & $75.90 \%$ & 7 & $24.10 \%$ & 29 & $100.00 \%$ \\
\hline & Total & 57 & $86.40 \%$ & 9 & $13.60 \%$ & 66 & $100.00 \%$ \\
\hline
\end{tabular}

Nota: OLQ $=$ Cuestionario de Orientación a la Vida

\begin{tabular}{|c|c|c|c|}
\hline Correlación Bivariada & & $\begin{array}{l}\text { ¿Qué ha sido lo más } \\
\text { importante para usted? }\end{array}$ & OLQ \\
\hline \multirow[t]{2}{*}{$\begin{array}{l}\text { ¿Qué ha sido lo más importante } \\
\text { para usted? }\end{array}$} & $\begin{array}{l}\text { Pearson } \\
\text { Correlation }\end{array}$ & 1 & $.303 *$ \\
\hline & $\mathrm{N}$ & 66 & 66 \\
\hline \multirow[t]{2}{*}{ OLQ } & $\begin{array}{l}\text { Pearson } \\
\text { Correlation }\end{array}$ & $.303^{*}$ & 1 \\
\hline & $\mathrm{N}$ & 66 & 67 \\
\hline
\end{tabular}

Nota: ${ }^{*} \mathrm{p}<.05$ (dos colas)

Si bien la relación entre dos variables discretas es difícil de interpretar, la tabla 5 sugiere que si existe algún tipo de asociación en el puntaje obtenido en el OLQ y aquello que es más importante para la persona. 
Tabla 6

Tablas de contingencia y correlación Pearson entre caso-no caso probable psiquiátrico y edad

\begin{tabular}{clcc|cc|cc}
\hline & \multicolumn{9}{c}{ CGS } \\
\hline & & \multicolumn{2}{c}{ No caso } & \multicolumn{2}{c}{ Caso } & \multicolumn{2}{c}{ Total } \\
\cline { 3 - 8 } & $n$ & $\%$ & $n$ & $\%$ & $n$ & $\%$ \\
\hline \multirow{4}{*}{ Edad } & 10 & 83.30 & 2 & 16.70 & 12 & 100.00 \\
& 60-64 años & 25 & 96.20 & 1 & 3.80 & 26 & 100.00 \\
& 65-69 años & 19 & 95.00 & 1 & 5.00 & 20 & 100.00 \\
& $70-74$ años & 3 & 60.00 & 2 & 40.00 & 5 & 100.00 \\
& $75-80$ años & 4 & 100.00 & 0 & 0.00 & 4 & 100.00 \\
& 80+ años & 61 & 91.00 & 6 & 9.00 & 67 & 100.00 \\
\hline
\end{tabular}

Nota $:$ CGS $=$ Cuestionario de Salud General

\begin{tabular}{llcc} 
& Correlación Bivariada & Edad & CGS \\
\hline \multirow{2}{*}{ Edad } & Pearson Correlation & 1 & $.016^{\mathrm{a}}$ \\
& $\mathrm{N}$ & 67 & 67 \\
\multirow{2}{*}{$\mathrm{CGS}$} & Pearson Correlation & $.016^{\mathrm{a}}$ & 1 \\
& $\mathrm{~N}$ & 67 & 67 \\
\hline \multicolumn{2}{c}{ Nota: ${ }^{\mathrm{a}} \mathrm{p}>.05$} &
\end{tabular}

Según se observa en la tabla 6, no existe correlación significativa entre la edad y ser o no caso probable psiquiátrico.

Tabla 7

Tablas de contingencia y correlación Pearson entre caso-no caso probable psiquiátrico y sexo

\begin{tabular}{|c|c|c|c|c|c|c|c|}
\hline \multirow{6}{*}{ Sexo } & & \multicolumn{6}{|c|}{ CGS } \\
\hline & & \multicolumn{2}{|c|}{ No caso } & \multicolumn{2}{|c|}{ Caso } & \multicolumn{2}{|c|}{ Total } \\
\hline & & $n$ & $\%$ & $n$ & $\%$ & $n$ & $\%$ \\
\hline & Masculino & 17 & 89.50 & 2 & 10.50 & 19 & 100.00 \\
\hline & Femenino & 44 & 91.70 & 4 & 8.30 & 48 & 100.00 \\
\hline & Total & 61 & 91.00 & 6 & 9.00 & 67 & 100.00 \\
\hline
\end{tabular}

Nota: CGS = Cuestionario de Salud General

(continúa) 
Tabla 7 (continuación)

\begin{tabular}{llcc} 
& Correlaciones & Sexo & CGS \\
\hline Sexo & Pearson Correlation & 1 & -.035 \\
& $\mathrm{~N}$ & 67 & 67 \\
CGS & Pearson Correlation & -.035 & 1 \\
& $\mathrm{~N}$ & 67 & 67 \\
\hline
\end{tabular}

En el caso de la tabla 7, los análisis sugieren que no existe relación entre el sexo y la variable caso-no caso probable psiquiátrico.

\section{Tabla 8}

Tablas de contingencia y correlación Pearson entre caso-no caso probable psiquiátrico y número de enfermedades

\begin{tabular}{|c|c|c|c|c|c|c|c|}
\hline \multicolumn{8}{|c|}{ CGS } \\
\hline & & \multicolumn{2}{|c|}{ No caso } & \multicolumn{2}{|c|}{ Caso } & \multicolumn{2}{|c|}{ Total } \\
\hline \multirow{7}{*}{$\begin{array}{l}\text { Número de } \\
\text { enfermedades }\end{array}$} & & $n$ & $\%$ & $n$ & $\%$ & $n$ & $\%$ \\
\hline & Ninguna & 42 & 95.50 & 2 & 4.50 & 44 & 100.00 \\
\hline & Una & 16 & 88.90 & 2 & 11.10 & 18 & 100.00 \\
\hline & Dos & 2 & 66.70 & 1 & 33.30 & 3 & 100.00 \\
\hline & Tres & 1 & 100.00 & 0 & 0.00 & 1 & 100.00 \\
\hline & Cuatro & 0 & 0.00 & 1 & 100.00 & 1 & 100.00 \\
\hline & Total & 61 & 91.00 & 6 & 9.00 & 67 & 100.00 \\
\hline
\end{tabular}

Nota $:$ CGS = Cuestionario de Salud General

\begin{tabular}{|c|c|c|c|}
\hline \multicolumn{2}{|c|}{ Correlaciones } & Número de enfermedades & CGS \\
\hline \multirow[t]{2}{*}{$\begin{array}{l}\text { Número de } \\
\text { enfermedades }\end{array}$} & $\begin{array}{l}\text { Pearson } \\
\text { Correlation }\end{array}$ & 1 & $.351 * *$ \\
\hline & $\mathrm{N}$ & 67 & 67 \\
\hline \multirow[t]{2}{*}{ CGS } & $\begin{array}{l}\text { Pearson } \\
\text { Correlation }\end{array}$ & $.351 * *$ & 1 \\
\hline & $\mathrm{N}$ & 67 & 67 \\
\hline
\end{tabular}

En el caso de la tabla 8, se observa una correlación positiva y significativa entre el número de enfermedades y la variable caso-no caso probable psiquiátrico. Esta relación es más visible entre el grupo no-caso y una menor frecuencia de enfermedades. 
Tabla 9

Tablas de contingencia y correlación Pearson entre caso-no caso probable psiquiátrico y lo qué ha sido más importante en la vida de los entrevistados

\begin{tabular}{clcc|cc|cc}
\hline & \multicolumn{3}{c}{ CGS } & \multicolumn{2}{c}{ Total } \\
\cline { 2 - 9 } & \multicolumn{2}{c}{ No caso } & $\%$ & $n$ & $\%$ & $n$ & $\%$ \\
\hline \multirow{2}{*}{$\begin{array}{c}\text { ¿Qué ha sido lo } \\
\text { más importante } \\
\text { para usted? }\end{array}$} & Situación afectiva & 32 & $94.10 \%$ & 2 & $5.90 \%$ & 34 & $100.00 \%$ \\
& Situación económica & 3 & $100.00 \%$ & 0 & $0.00 \%$ & 3 & $100.00 \%$ \\
& Su salud & 25 & $86.20 \%$ & 4 & $13.80 \%$ & 29 & $100.00 \%$ \\
& Total & 60 & $90.90 \%$ & 6 & $9.10 \%$ & 66 & $100.00 \%$ \\
\hline
\end{tabular}

Nota: CGS $=$ Cuestionario de Salud General

¿Qué ha sido lo más
importante para usted? $\quad$ CGS

\begin{tabular}{llcc}
\hline $\begin{array}{l}\text { ¿Qué ha sido lo más } \\
\text { importante para usted? }\end{array}$ & $\begin{array}{l}\text { Pearson } \\
\text { Correlation }\end{array}$ & 1 & .133 \\
& $\mathrm{~N}$ & 66 & 66 \\
CGS & $\begin{array}{l}\text { Pearson } \\
\text { Correlation }\end{array}$ & .133 & 1 \\
& $\mathrm{~N}$ & 66 & 67 \\
\hline
\end{tabular}

Finalmente, la tabla 9 revela que no existe relación significancia entre ser o no caso probable psiquiátrico y el lugar que ocupan la salud y el aspecto afectivo y económico en las vidas de los entrevistados. 


\section{Discusión}

Este estudio muestra resultados alentadores para la población adulta mayor, llamado así el grupo etario que sobrepasa los 60 años de edad, ya que la muestra entrevistada tuvo puntajes elevados en el instrumento que mide sentido de coherencia. También se encontró una proporción relativamente baja de casos probables psiquiátricos.

Es de mencionarse que todas las personas entrevistadas percibían su salud como buena o muy buena en el momento del estudio. Se hace necesario aclarar que una proporción minoritaria de ellos padecía una o más enfermedades, aunque, padecerlas no afectó la autopercepción positiva sobre su salud.

Las personas de la muestra se desenvuelven gran parte del día en un ambiente favorable para su estado de ánimo, este lugar es una estancia diurna diseñada y dedicada especialmente para ellos, la gran mayoría realiza algún tipo de deporte y convive diariamente con personas de su edad, lo cual, además de dotarlos de un alto SOC, probablemente ha contribuido a afianzar y/o desarrollar sus capacidades y habilidades con relación a las oportunidades que el medio les presenta y les ofrece la posibilidad de cubrir y plantear nuevas expectativas para su desarrollo y contribuir a su bienestar material y subjetivo.

Todo este capital acumulable a lo largo de la vida del adulto mayor incorpora herramientas o habilidades aprendidas que se confrontan continuamente con los retos de la vida cotidiana para promover y mantener el bienestar subjetivo al que muchos se refieren cuando responden que tienen una buena o muy buena salud.

Argyle (1992) hace referencia a la importancia de la capacidad individual para enfrentarse a los problemas de la vida y que desemboca en un estado de felicidad. La percepción de las capacidades se engarza en un marco de creencias y valores que proporcionan una identidad cultural y producen satisfacción y felicidad, es decir, bienestar subjetivo.

Herrán de la y Arita (2003) y Arita y Herrán de la (2007) analizan la creencia en la capacidad en un plano psicosocial en cuanto a la salud, educación, estatus social, seguridad personal y nivel cultural percibidos por el individuo. A su vez, Cummins (2000, 2004), Cummins, Dzuka, y Arita (2003), Cummins y Lan (2006) desarrollaron la teoría homeostática del bienestar personal, proponiendo que los mecanismos psicológicos internos actúan automáticamente para mantener un sentido de bienestar personal, prediciendo además que la satisfacción personal por la vida evaluada de manera general será alta y relativamente estable.

Además, a esta edad, el componente psicológico desempeña un factor importantísimo en la salud, pues los añosos, en algunos casos, tienen alguna enfermedad de tipo crónico degenerativo, lo que no influye en seguirse percibiendo como sanos, lo más importante y lo que es la salud para ellos, es el grado de funcionalidad que tienen, la capacidad de disfrute que poseen, la manera de relacionarse con los demás y su satisfacción por la vida en general.

Se encontró en este estudio que las personas con un nivel alto de sentido de coherencia, significativamente, lo más importante y por encima de su salud ha sido el aspecto afectivo, lo cual quiere decir que a esta edad también el componente afectivo influye decisivamente en la autopercepción sobre la salud, así, una persona satisfecha y feliz tiende más a percibirse como sana que una insatisfecha.

La edad no fue un factor influyente en la autopercepción de la salud ni en el nivel de SOC, $\mathrm{y}$, dato curioso, tampoco el sexo influyó, tanto hombres como mujeres tuvieron niveles altos de SOC, siendo que, diversos estudios puntúan a la mujer con mucho más presencia de enfermedades 
que los hombres en todas las etapas de su vida (Sojo, Sierra, \& López, 2002). Tampoco influyó el sexo ni la edad en el estado de salud mental.

A partir de estos hallazgos puede detectarse la necesidad de atender los elementos psicológicos y socio afectivos que integran la salud de una persona mayor, considerando que cada ser es único y que lo que satisface y es saludable para uno puede no ser válido para otro. De aquí surge la recomendación de investigar qué satisface a una persona mayor, qué le da mayor placer en la vida y tratar de satisfacerlo sin perjudicarla en ningún aspecto. Desde luego, sin olvidar reafirmar la necesidad de llevar estilos de vida sanos donde se incluya el ejercicio y la alimentación adecuados.

La actual preocupación que la sociedad muestra por la salud puede llevar a caer en el error de considerarla más como un fin (alcanzar ese bienestar utópico) que como un medio (vivir encontrándose bien). En cierta medida, los medios de comunicación y las tendencias estéticas del culto al cuerpo, bajo las que subyacen sabrosos intereses económicos, parecen orientar el logro de la salud como si de una meta se tratara. El peligro de esta concepción estriba en identificar la adopción de hábitos saludables como un sacrificio que obliga a las personas a renunciar a muchos placeres de la vida que creen insalubres. El afán por vivir saludablemente no debe implicar el divorcio entre salud y calidad de vida.

En este sentido, la idea fundamental que dimana de la promoción de la salud es que su objetivo último, más que prolongar los años de vida, lo que pretende es que se vivan mejor.

Cuando se dice que el objetivo es que los últimos años se vivan mejor, se habla de vivirlos saludablemente, es decir con salud; en este sentido, la salud es un estado de equilibrio, con determinado grado de componente subjetivo, entre lo biológico y lo psíquico con el medio ambiente social, cultural y natural. Así, pues, la salud no significa simplemente ausencia de enfermedad, es un estado biocultural de equilibrio relativo y función normal mantenidos dentro de contextos temporales, sociales, culturales y ecológicos específicos.

\section{Referencias}

Aburto, C. (2001). Estilos de vida y salud: su efecto en la mortalidad. Psicología y Salud,11(2), 543-559.

Antonovsky, A. (1979). Health, stress and coping: New perspectives on mental and physical wellbeing. San Francisco: Jossey-Bass.

Antonovsky, A. (1987). Unraveling the mystery of health. How people manage stress and stay well. San Francisco: Jossey-Bass.

Antonovsky, A. (1992). Can attitudes contribute to health? Advances, 8, 33-49.

Argyle, M. (1992). La psicología de la felicidad. Madrid: Alianza Editorial.

Arita, B. (2006). Calidad de vida en Culiacán. Condiciones objetivas, capacidad y bienestar subjetivos. México: Fontamara (Colección ABC): Sinaloa.

Arita, B. \& Herrán de la, J. (2007). El papel de la Universidad y a educación en la formación de capacidades para la vida. Ponencia presentada en el VIII Congreso Nacional y IV Congreso Internacional de la Red de Investigación y Docencia sobre Innovación Tecnológica. Culiacán, Sinaloa.

Barra, E. (2002). Psicología de la salud. Santiago de Chile: Universidad de Concepción. Belsky, J. (2001). Psicología del envejecimiento. Madrid: Paraninfo. 
Birren, J. (1996). Autobiography: Exploring the self, and encouraging development. En K. Gary \& R. Jan-Erik (Eds): Aging and Biography: Explorations in adult development (pp. 180-210). New York: Springer.

Brizzio, A. \& Carreras, A. (2007). Variables salutogénicas y su relación con los sucesos de vida. Revista Iberoamericana de Diagnóstico y Evaluación Psicológica, 23(1), 83-99.

Caraveo, A., González, F., \& Ramos, L. (1985) Indicadores clínicos de alteración psiquiátrica en la práctica médica general. Salud Pública, 27, 140-141.

Carmel, S., Anson, O., Levenson, A., Bonneth, D. Y., \& Maoz, B. C. (1991). Life events, sense of coherence and health: gender differences on the kibbutz. Social Science and Medicine, 32, 1089-1096.

Casullo, M. (2008). Prácticas en psicología positiva. Buenos Aires: Lugar Editorial.

Coe, R., Romers, J., Tang, B., \& Wolinsky, F. (1990). Correlates of a measure of coping in older veterans: A preliminary report. Journal of Community Health, 15(5), 287-296.

Coolican, H. 1994. Métodos de investigación y estadísticos en psicología. México: El Manual Moderno.

Cornachione, M. (2006). Psicología del desarrollo. Aspectos biológicos, psicológicos y sociales. Buenos Aires: Editorial Brujas.

Cummins, R. (2000). Objective and subjective quality of life: An interactive model. Social Indicators Research (Ámsterdam), 52, 55-72.

Cummins, R. (2004). The international well-being: A psychometric progress report. Ponencia presentada en la Sexta Conferencia de la Sociedad Internacional para el Estudio de la Calidad de Vida (ISQOLS). Frankfurt. Universidad de Frankfurt.

Cummins, R., Dzuka, J., \& Arita, B. (2003). A comparison of the personal Well-being Index in Slovakia, México and Australia. Documento presentado en la Séptima Conferencia de la Sociedad, Internacional para el estudio de la Calidad de Vida (ISQOLS). Grahamstown (Sudáfrica): Rhodes University.

Cummins, R. \& Lan, A. (2006). The personal Well-being Index as one of four theoretically linked measures of subjective well-being. Documento presentado en la Séptima Conferencia de la Sociedad Internacional para el Estudio de la Calidad de Vida (ISQOLS). Grahamstown (Sudáfrica): Rhodes University.

Diener, E. \& Suh, E.M. (2001). Culture and subjective well-being. Cambridge MA: The Mit Press

Duchan, J. \& Black, S.E. (2001). Progressing toward life goals: a person-centered approach to evaluating therapy. Topics in Language Disorders, 22(1), 37-49.

Ezban, B., Padilla, G., Medina, M., \& Gutiérrez, C. (1985). Aplicación de un cuestionario de detección de casos psiquiátricos en dos poblaciones de la práctica médica general. Salud Pública de México, 27, 384-390.

Gallard, M. (2002). Promoción de la salud y apoyo psicológico al paciente. Madrid: Thomson Paraninfo.

Gil-Monte, P. \& Peiró, J.M. (1997). Estrategias de intervención para la prevención y afrontamiento del síndrome de quemarse por el trabajo. En: P. Gil-Monte \& J.M. Pierrot (Eds.). El desgaste psíquico en el trabajo: El síndrome de quemarse. Madrid: Síntesis.

Giraldo, A., Toro, M., Macías, A., Valencia, C., \& Palacios, S. (2010). La promoción de la salud como estrategia para el fomento de estilos de vida saludables. Hacia la Promoción de la Salud, 15(1), 128-143. 
Herrán de la, J. \& Arita, B (2003). Escala de capacidades. Documento interno. Culiacán, Sinaloa. (México): Universidad Autónoma de Sinaloa.

Lazarus, R. \& Folkman, S. (1991). Estrés y procesos cognitivos. México: Roca.

Margalit, M., Leyser, Y., \& Avraham, Y. (1988). Subtypes of family climate among kibbutz mothers of disabled children. International Journal of Special Education, 3, 101-115.

Mc Dowell, I. \& Newell, C. (1987). Measuring Health. New York: Oxford University Press.

Meda, R., Torres, P., Cano, R., \& Vargas, R. 2004. Creencias de salud-enfermedad y estilos de vida en estudiantes universitarios. Psicología y Salud, 14(2), 205-214.

Medina, M., Padilla, G., \& Ezban, B. (1983) The factor structure of the G.H.Q. a escaled version for a Hospital General Practice service in México. Psychologycal Medicine, 13, 355-361.

Muchinik, E. (2006). Envejecer en el siglo XXI. Buenos Aires: Lugar Editorial.

Naughton, M.J. \& Wiklund, I. (1993). A critical review of dimension-specific measures of healthrelated quality of life in cross-cultural research. Quality of Life Research, 2, 385-401.

Radmacher, S. \& Sheridan, C. (1989). The global inventory of stress. A comprehensive approach to stress assessment. Medical Psychotherapy, 2, 183-188.

Romero, M. \& Medina, M. (1987). Validez de una versión del Cuestionario General de Salud para detectar psicopatología en estudiantes universitarios. Salud Mental, 10, 196-203.

Sen, A. (2004). Capital humano y capacidad humana. Foro de Economía Política. Recuperado de http://www.red-vértice.com.

Sojo, D., Sierra, B., \& López, I. (2002). Salud y Género. Madrid: Médicos del Mundo.

Suárez, C., Del Toro, M. Moncada, C. Vinent, M., \& Peña, M. (2001). La capacidad de aprendizaje en la formación integral del estudiante. La Habana: Centro de Estudios de la Educación Superior de la Universidad de Oriente.

\section{Reseña de las autoras}

\section{Zoila Edith Hernández Zamora}

Grado Académico: Doctora. Especialidad: Educación. Institución en donde se graduó: Universidad LaSalle de Costa Rica en convenio con la Universidad Veracruzana, México. Institución en a que trabaja y puesto actual: Instituto de Investigaciones Psicológicas de la Universidad Veracruzana. Investigadora.

\section{Yamilet Ehrenzweig Sánchez}

Grado Académico: Doctora. Especialidad: Educación. Institución en donde se graduó: Universidad LaSalle de Costa Rica en convenio con la Universidad Veracruzana, México. Institución en a que trabaja y puesto actual: Instituto de Investigaciones Psicológicas de la Universidad Veracruzana. Investigadora.

\section{Liliana Yépez Olvera}

Grado Académico: Licenciatura. Especialidad: Psicología. Institución en donde se graduó: Facultad de Psicología de la Universidad Veracruzana, México. Institución en la que trabaja y puesto actual: Instituto de Investigaciones Psicológicas. Becaria. 